\title{
Association of participation in a supplemental nutrition program with stillbirth by race, ethnicity, and maternal characteristics
}

\author{
Meghan Angley ${ }^{1 *}$ D, Vanessa R. Thorsten², Carolyn Drews-Botsch, Donald J. Dudley ${ }^{3}$, Robert L. Goldenberg ${ }^{4}$, \\ Robert M. Silver ${ }^{5}$, Barbara J. Stoll ${ }^{6}$, Halit Pinar ${ }^{7}$ and Carol J. R. Hogue ${ }^{1}$
}

\begin{abstract}
Background: Participation in the Special Supplemental Nutrition Program for Women, Infants and Children (WIC) has been associated with lower risk of stillbirth. We hypothesized that such an association would differ by race/ ethnicity because of factors associated with WIC participation that confound the association.

Methods: We conducted a secondary analysis of the Stillbirth Collaborative Research Network's population-based case-control study of stillbirths and live-born controls, enrolled at delivery between March 2006 and September 2008. Weighting accounted for study design and differential consent. Five nested models using multivariable logistic regression examined whether the WIC participation/stillbirth associations were attenuated after sequential adjustment for sociodemographic, health, healthcare, socioeconomic, and behavioral factors. Models also included an interaction term for race/ethnicity $\times$ WIC.

Results: In the final model, WIC participation was associated with lower adjusted odds (aOR) of stillbirth among non-Hispanic Black women (aOR: 0.34; $95 \% \mathrm{Cl} 0.16,0.72$ ) but not among non-Hispanic White (aOR: 1.69; 95\% Cl: 0.89, 3.20) or Hispanic women (aOR: 0.91; 95\% Cl 0.52, 1.52).

Conclusions: Contrary to our hypotheses, control for potential confounding factors did not explain disparate findings by race/ethnicity. Rather, WIC may be most beneficial to women with the greatest risk factors for stillbirth. WIC-eligible, higher-risk women who do not participate may be missing the potential health associated benefits afforded by WIC.
\end{abstract}

Keywords: Stillbirth, Racial disparities, WIC

\section{Background}

The Special Supplemental Nutrition Program for Women, Infants and Children (WIC), established in the 1970's in the United States, provides assistance in purchasing nutritious foods to pregnant and postpartum women and infants and children up to age five [1]. While there is a long history of research on WIC participation and its association with birth outcomes, study results are mixed, and benefits of WIC may be overstated due to challenges

\footnotetext{
* Correspondence: mangley@emory.edu

${ }^{1}$ Department of Epidemiology, Rollins School of Public Health, Emory

University, 1518 Clifton Road, Atlanta, GA 30322, USA

Full list of author information is available at the end of the article
}

associated with non-random prenatal participation in WIC and gestational age bias [2-4].

Non-random participation of women in WIC is a challenge in assessing the positive impact of WIC on birth outcomes because it is estimated that only two thirds of eligible pregnant or postpartum women participate in WIC [5]. Eligible Black and Hispanic women are more likely to participate than eligible White women. WIC participation is negatively correlated with education, and participation is correlated with non-married status [5]. Further, WIC enrollment criteria stipulate that women be at "nutritional risk," which includes overweight and underweight women, women with certain conditions,

(c) The Author(s). 2018 Open Access This article is distributed under the terms of the Creative Commons Attribution 4.0 International License (http://creativecommons.org/licenses/by/4.0/), which permits unrestricted use, distribution, and 
such as diabetes or hypertension, and women with prior adverse pregnancy outcomes [6]. Nearly $70 \%$ of pregnant women participating in WIC have more than one nutritional risk factor [1]. Women who choose to participate in WIC may be more health conscious and/or more motivated to prevent adverse outcomes than WIC eligible women who choose not to participate [2]. That said, WIC participation is also associated with smoking during pregnancy among some women [7].

Several recent studies have attempted to address these limitations. Sonchak et al. [8] attempted to control for non-random participation in WIC by examining women who change their WIC status over their reproductive life course. These investigators found WIC to be associated with increased average birth weight and length of gestation and reduced percentage of infants with low birth weight and neonatal intensive care unit admission [8]. Fingar et al. [9], using a fetuses-at-risk approach to address gestational age bias, found that WIC enrollment by gestational week 29 was associated with a lower risk of preterm birth, low birth weight and perinatal death.

Despite conflicting conclusions regarding the overall relationship between WIC and birth outcomes, a consistent finding is that if identified, benefits of WIC are more pronounced among high-risk women. In a study by Sonchak et al., after controlling for gestational age, the association between WIC and lower risk of low birth weight was significant only for Black women [8]. In another study, WIC was protective against fetal death among women with low education and against preterm birth among women with inadequate prenatal care [10]. WIC participation in Kansas was associated with reduced infant mortality among Black and Hispanic, but not White women [11].

While there is some evidence linking WIC to a reduction in fetal death $[9,10]$, few studies examined racial differences in the association between WIC and risk of stillbirth. In the United States, racial disparities in stillbirth rates persist, with the rate of stillbirths among African American mothers at 10.53 per 1000 live births and stillbirths, compared to 4.88 among White women and 5.22 among Hispanic women in 2013 [12]. Maternal sociodemographic characteristics and nutritional eligibility criteria are associated with stillbirth risk [13], and these factors could also contribute to the observed effect of WIC participation on stillbirth.

The initial objective of our study was to investigate whether the characteristics of women who participate in WIC during pregnancy differ by race or ethnicity. Characteristics examined included comorbid conditions that may affect stillbirth risk, health care utilization during pregnancy, sociodemographic factors and substance use. We then examined if the association between WIC and stillbirth was modified by race or ethnicity and if so, if these differences could be explained by factors associated with participation in WIC. We hypothesized that racial/ethnic differences in the association between WIC and stillbirth would be eliminated by accounting for factors associated with participation in WIC.

\section{Methods}

\section{Data source}

We used data from the Stillbirth Collaborative Research Network (SCRN), a population-based case-control study of stillbirths and live-born controls enrolled at the time of delivery. The design and methods of the study have been previously described [14]. Resident women were enrolled in five selected geographically defined areas (Bristol County, Massachusetts and the state of Rhode Island; DeKalb County, Georgia; Galveston and Brazoria Counties, Texas; Bexar County, Texas and Salt Lake County, Utah) and who delivered between March 2006 and September 2008 at 59 hospitals chosen to capture at least $90 \%$ of the resident deliveries. All stillbirths at each site occurring at 18 weeks of gestation or later were selected to be approached for participation in the study. A fetus was considered stillborn if Apgar scores were 0 at 1 and $5 \mathrm{~min}$ and there were no signs of life by direct observation. Stillbirths occurring at 18 weeks of delivery up to 20 weeks of delivery were eligible only if they met study criteria and gestational ages were not well dated.

Study personnel screened and registered all livebirths occurring at 20-31 weeks gestation and attempted to enroll controls with pre-defined selection probabilities by week of gestational age in order to enroll adequate numbers of controls born at early gestational ages. For livebirths occurring at greater than or equal to 32 weeks gestational age, random hospital-date-times were selected for each site, and the next eligible delivery in each selected time window was invited to participate. Study personnel gained the permission of women's physicians before approaching them regarding the study.

Women completed detailed maternal interviews on demographic, behavioral and psychosocial factors. Interviews were largely completed during women's delivery hospitalizations, but it was possible for women to complete the second half of the interview 2-4 weeks after delivery by phone. When available, medical records were abstracted for information on prenatal care, hospitalizations during pregnancy and the delivery. The study was approved by the institutional review boards of the academic centers, the participating hospitals and the data coordinating center. All participants in the study provided written informed consent.

This secondary analysis was restricted to self-identified non-Hispanic White, non-Hispanic Black and Hispanic women with singleton gestations who completed the maternal interview and for whom medical records were 
abstracted. Throughout this paper, we refer to these groups as White, Black, and Hispanic.

All demographic information came from the maternal interview. Women were categorized as having chronic hypertension and pre-existing diabetes if the conditions were noted in their chart and/or the women self-reported having these conditions. Pre-pregnancy body mass index (BMI) was determined using the woman's height and weight recorded in prenatal care medical records. Women with a BMI greater than or equal to $30 \mathrm{~kg} / \mathrm{m}^{2}$ were considered obese. Trimester of the start of prenatal care was captured from women's self-reported number of days between becoming pregnant and initiating prenatal care, since information solely from chart abstraction data could misclassify women as starting prenatal care later if chart data from their first prenatal care visit could not be located. Hospitalization prior to the delivery hospitalization was captured by noting any records of hospitalization from chart abstraction.

\section{Statistical Analyses.}

We first examined participation in WIC among stillbirths and live births by maternal characteristics, stratified by race/ethnicity. In addition, to assess the possible impact of gestational age bias, we also examined how gestational age at delivery was related to WIC participation in each race/ ethnicity group by using logistic regression to model WIC participation as a function of stillbirth status, controlling for gestational age as a continuous variable. Next, we constructed five nested models to assess the association between WIC participation and stillbirth by race and ethnicity to examine if the relationships were attenuated after adjustment for each set of covariates that have been associated with WIC participation in previous studies. The models were constructed to account sequentially for several categories of factors: 1) factors proximally associated with both stillbirth risk and WIC participation, 2) comorbid conditions, 3) health-care utilization, 4) socio-economic variables and 5) substance use, including smoking and drug use. The five models were as follows:

- Model 1: Adjusted for maternal age, insurance status, gestational age at delivery and pregnancy history.

- Model 2: Adjusted for all variables in Model 1, preexisting diabetes, hypertension and prepregnancy obesity.

- Model 3: Adjusted for all variables in Model 2, trimester of entry into prenatal care and chartdocumented hospitalizations during pregnancy.

- Model 4: Adjusted for all variables in Model 3, marital status, receipt of wages (by any household member) and education.
- Model 5: Adjusted for all variables in Model 4, smoking status during pregnancy and lifetime illicit drug use.

We first ran the models stratified by race and ethnicity. For each of these models, we noted the total number of observations, by case-control status and by race that we included after dropping observations with missing data. Second, we examined the association between WIC participation and stillbirth with an interaction term for race/ethnicity $\mathrm{x}$ WIC to assess differences in the relationship by race/ethnicity. Using these models, we examined both the association between WIC and stillbirth, using the 'effects' statement in SAS-Callable SUDAAN to generate race-specific odds ratios and 95\% confidence intervals for the association between WIC and stillbirth, as well as the association between race/ethnicity and stillbirth while controlling for all other variables. For all models, we used multivariable logistic regression with generalized estimating equations to account for nine women enrolled twice for two different pregnancies. All models also controlled for catchment area.

We also conducted several sensitivity analyses. First, to address the fact that we did not have data on WIC eligibility in our data, we examined the final model (Model 5) restricted to women who might be eligible for WIC, specifically women who reported having Medicaid or no insurance (Sensitivity A). Next, to examine if the observed associations were robust after restricting to a subset of women with similar prior pregnancy histories, we ran Model 5 restricted to nulliparous women who had experienced no previous pregnancy losses (Sensitivity B). We controlled for gestational age at delivery in Models $1-5$, but to remove gestational age as a potential consideration, we restricted Model 5 to deliveries occurring at 37 weeks' gestation or later without further controlling for gestational age. Furthermore, it has been noted that without controlling for gestational age in a multivariable analysis, it can approximate a fetuses-at-risk approach [15]. Therefore, we also ran Models 1-5 without controlling for gestational age. All statistical analyses were weighted to account for study sampling and differential consent. Analyses were conducted using SAS-Callable SUDAAN software Version 11.0.1 (Research Triangle Institute, Research Triangle Park, NC).

\section{Results}

A weighted sample of 1229 live births and 538 stillbirths was included in this analysis. Among White, Black and Hispanic women with live born infants, 20, 53 and 52\%, respectively participated in WIC. Among White, Black and Hispanic women with stillborn infants, 23, 35 and 
$47 \%$, respectively participated in WIC. We did not have data available for the timing of initiating WIC.

Across all racial/ethnic groups, younger women were more likely to be enrolled in WIC compared with older women (Additional file 1). Among women with chronic hypertension, diabetes or obesity, White women were less likely to participate in WIC than Black and Hispanic women, though these conditions themselves were not associated with WIC participation within racial/ethnic groups. Black and Hispanic women who were married, received household wages or had 13 or more years of education were more likely to be enrolled in WIC than their White counterparts.

Gestational age was most prominently associated with WIC participation among White women. Among live births to White women, a greater proportion of women with deliveries at 32 weeks or later participated in WIC, compared to women with deliveries earlier than 32 weeks. Also among White women, stillborn status was associated with WIC enrollment (OR: 2.23; 95\% CI: $1.23,3.96)$ while controlling for gestational age, but this was not true among Black or Hispanic women (Table 1).

In the unadjusted model stratified by race, the strongest association between WIC and stillbirth was among Black women, with reduced odds of stillbirth (OR: 0.49; 95\% CI: $0.31,0.77)$. Across the five nested models stratified by race, WIC participation was associated with reduced odds of stillbirth among Black women and was not associated with stillbirth among Hispanic women (Table 2). Adjustment across all models sequentially reduced the elevated risk of stillbirth for White women enrolled in WIC, but had little effect on the odds ratios for either Black or Hispanic women (Table 2). When all observations are included in the same models with an interaction term for maternal race/ethnicity $\mathrm{x}$ WIC on the odds of stillbirth, similar trends are apparent (Table 3). There appears to be no association between WIC participation and stillbirth among Hispanic women, while WIC participation was associated with reduced odds of stillbirth among Black women across all five models, and greater odds of stillbirth among White women, though the association was attenuated and became imprecise as variables were added to the models. In the final model (Model 5), WIC participation was significantly associated with lower odds of stillbirth among Black women (aOR: 0.34; 95\% CI 0.16, 0.72 ) but not White (aOR: $1.69 ; 95 \%$ CI: $0.89,3.20)$ or Hispanic women (aOR: 0.91; 95\% CI 0.52, 1.52) (Table 3).

Using these same models, associations between race and stillbirth for both women not on WIC and women participating in WIC are depicted in Table 4, again using the 'effects' statement to generate odds ratios and 95\% confidence intervals. Among women not receiving WIC, there were consistently higher odds of stillbirth for Black and for Hispanic women in Models 1-3 compared to White women. Among women receiving WIC during pregnancy, the odds ratios for the association of maternal race and stillbirth were imprecise, but were not significantly elevated among Black and Hispanic women compared to White women.

Restricting Model 5 in Table 3 to term deliveries did not change the direction and magnitude of the relationships. Not controlling for gestational age in Models 1-5 did not change the fact that WIC appeared protective among Black women in all Models, but among White women, the associations were pulled to the null. For example, an odds ratio of 1.48 (95\% CI: $0.95,2.30)$ compared to an odds ratio of 2.23 (95\% CI: 1.22, 4.10) in Table 3, Model 1. In addition, restricting Model 5 to women who are most likely to be eligible for WIC (Sensitivity A) showed similar trends, with WIC being associated with reduced odds of stillbirth among Black women and no association among White or Hispanic women (Tables 2-3). However, when Model 5 was restricted to nulliparous women with no previous losses, the racial/ ethnic differences in the association between WIC and stillbirth were eliminated, with the respective adjusted odds ratios for White, Black and Hispanic women as 1.00 (95\% CI 0.33, 3.02), 0.86 (95\% CI $0.22,3.33$ ) and 0.96 (95\% CI 0.34, 2.67) (Table 3).

\section{Discussion}

Our findings are consistent with studies of WIC participation and outcomes other than stillbirth, in which positive outcomes appear more pronounced among Black women enrolled in WIC compared to other racial/ethnic groups $[8,11]$. This racial/ethnic difference in outcome

Table 1 Proportion of women reporting enrollment in WIC by race/ethnicity, gestational age and stillbirth status

\begin{tabular}{|c|c|c|c|c|c|c|c|c|c|c|c|c|}
\hline \multirow[b]{2}{*}{ Gestational Age } & \multicolumn{2}{|c|}{$\begin{array}{l}\text { White Live } \\
\text { Born }(N=641)^{\mathrm{b}}\end{array}$} & \multicolumn{2}{|c|}{$\begin{array}{l}\text { White Stillborn } \\
(N=205)^{b}\end{array}$} & \multicolumn{2}{|c|}{$\begin{array}{l}\text { Black Live } \\
\text { Born }(N=331)^{b}\end{array}$} & \multicolumn{2}{|c|}{$\begin{array}{l}\text { Black Stillborn } \\
(N=123)^{b}\end{array}$} & \multicolumn{2}{|c|}{$\begin{array}{l}\text { Hispanic Live } \\
\text { Born }(N=666)^{b}\end{array}$} & \multicolumn{2}{|c|}{$\begin{array}{l}\text { Hispanic Stillborn } \\
(N=205)^{b}\end{array}$} \\
\hline & Weighted N & $\% \mathrm{WIC}$ & Weighted N & $\% \mathrm{WIC}$ & Weighted N & $\% \mathrm{WIC}$ & Weighted N & $\% \mathrm{WIC}$ & Weighted N & $\% \mathrm{WIC}$ & Weighted N & $\% \mathrm{WIC}$ \\
\hline$<32$ weeks & 7 & 15.2 & 113 & 16.8 & 5 & 49.7 & 102 & 34.9 & 10 & 44.9 & 123 & 44.3 \\
\hline $32+$ weeks & 589 & 19.6 & 80 & 32.7 & 147 & 52.7 & 32 & 34.0 & 471 & 52.1 & 88 & 50.0 \\
\hline $\begin{array}{l}\text { Odds Ratio } \\
(95 \% \text { Cl) }\end{array}$ & $\begin{array}{l}2.21 \\
(1.23,3.96)\end{array}$ & & & & $\begin{array}{l}0.58 \\
(0.29,1.16)\end{array}$ & & & & $\begin{array}{l}0.94 \\
(0.59,1.50)\end{array}$ & & & \\
\hline
\end{tabular}

adds ratio for WIC enrollment, as a function of stillborn/live born status controlling for gestational age in weeks (continuous)

${ }^{\mathrm{b}}$ Unweighted counts 
Table 2 Association between WIC participation and stillbirth, stratified by race

\begin{tabular}{|c|c|c|c|c|c|c|c|c|c|c|c|c|}
\hline \multirow[b]{2}{*}{ Model } & \multicolumn{4}{|c|}{ White non-Hispanic } & \multicolumn{4}{|c|}{ Black non-Hispanic } & \multicolumn{4}{|l|}{ Hispanic } \\
\hline & Live births $^{a}$ & Stillbirths $^{a}$ & OR & $95 \% \mathrm{Cl}$ & Live births $^{a}$ & Stillbirths ${ }^{a}$ & OR & $95 \% \mathrm{Cl}$ & Live births $^{a}$ & Stillbirths ${ }^{a}$ & OR & $95 \% \mathrm{Cl}$ \\
\hline Unadjusted $^{\mathrm{b}}$ & 593 & 191 & 1.33 & $0.87,2.02$ & 151 & 134 & 0.49 & $0.31,0.77$ & 480 & 211 & 0.84 & $0.60,1.16$ \\
\hline Model $1^{c}$ & 593 & 191 & 2.21 & $1.06,4.59$ & 151 & 130 & 0.32 & $0.15,0.69$ & 479 & 211 & 1.32 & $0.80,2.18$ \\
\hline Model 2 & 592 & 189 & 2.13 & $1.03,4.43$ & 147 & 130 & 0.33 & $0.15,0.71$ & 468 & 206 & 1.13 & $0.67,1.89$ \\
\hline Model 3 & 588 & 188 & 2.15 & $1.03,4.48$ & 147 & 128 & 0.35 & $0.16,0.78$ & 466 & 204 & 1.17 & $0.69,1.99$ \\
\hline Model 4 & 585 & 188 & 1.66 & $0.74,3.73$ & 147 & 126 & 0.33 & $0.15,0.74$ & 463 & 204 & 1.16 & $0.68,1.96$ \\
\hline Model 5 & 580 & 185 & 1.49 & $0.66,3.35$ & 147 & 123 & 0.31 & $0.14,0.68$ & 458 & 204 & 1.14 & $0.67,1.94$ \\
\hline Sensitivity $A^{d}$ & 161 & 52 & 2.11 & $0.75,5.95$ & 102 & 77 & 0.23 & $0.09,0.59$ & 286 & 133 & 2.16 & $1.06,4.37$ \\
\hline Sensitivity B & 199 & 65 & 0.22 & $0.06,0.85$ & 47 & 36 & 0.80 & $0.14,4.47$ & 115 & 79 & 0.99 & $0.33,2.92$ \\
\hline
\end{tabular}

${ }^{a}$ Weighted counts

bunadjusted associations control only for catchment area

c Model 1: Adjusted for maternal age, insurance status, gestational age at delivery and pregnancy history

Model 2: Adjusted for all variables in Model 1, preexisting diabetes, hypertension and pre-pregnancy obesity

Model 3: Adjusted for all variables in Model 2, trimester of entry into prenatal care and chart-documented hospitalizations during pregnancy

Model 4: Adjusted for all variables in Model 3, marital status, receipt of wages (by any household member) and education

Model 5: Adjusted for all variables in Model 4, smoking status during pregnancy and lifetime illicit drug use

${ }^{d}$ A: Model 5 restricted to women on Medicaid or without insurance

B: Model 5 restricted to nulliparous women who had experienced no previous pregnancy losses

by WIC status held true after controlling for factors that may be associated with participation in WIC. These relationships also remained consistent when restricting the sample to deliveries occurring at 37 weeks' gestation or later, restricting to women who had Medicaid or no insurance and not controlling for gestational age at delivery. However, when restricting to nulliparous women with no previous losses, racial/ethnic differences in the association between WIC and stillbirth were eliminated.

Although we controlled for a number of factors that may be associated with participation in WIC and confounders of the relationship between WIC and stillbirth, the observed relationship may be due to unmeasured confounders. Residual confounding may also differ between racial/ethnic groups. White women who enroll in WIC may have a disproportionate distribution of unmeasured risk factors for stillbirth. In a study of WIC-eligible women, higher income was associated with a decrease in WIC participation and unintended pregnancy was associated with increased participation in WIC only among White women [16]. This was also true in our population, where among live births to women who received household wages, only $17.2 \%$ of White women participated in WIC compared to $50 \%$ of their Black and Hispanic counterparts. Generally, families that are eligible but choose not to enroll in WIC have more economic and personal resources [17], but one possibility is that among White women these economic and personal resources do not translate to a reduced risk of stillbirth.

Khanani et al. also found that while the infant mortality rate was reduced among Black WIC participants

Table 3 Association between WIC and stillbirth by maternal race in models with interaction terms for WIC x maternal race

\begin{tabular}{|c|c|c|c|c|c|c|}
\hline \multirow[b]{2}{*}{ Model $^{a}$} & \multicolumn{2}{|c|}{ White non-Hispanic } & \multicolumn{2}{|c|}{ Black non-Hispanic } & \multicolumn{2}{|l|}{ Hispanic } \\
\hline & Odds Ratio & $95 \% \mathrm{Cl}$ & Odds Ratio & $95 \% \mathrm{Cl}$ & Odds Ratio & $95 \% \mathrm{Cl}$ \\
\hline Model 1 & 2.23 & $1.22,4.10$ & 0.32 & $0.15,0.67$ & 1.10 & $0.66,1.85$ \\
\hline Model 2 & 2.11 & $1.14,3.89$ & 0.32 & $0.15,0.66$ & 0.92 & $0.55,1.53$ \\
\hline Model 3 & 2.14 & $1.15,3.99$ & 0.32 & $0.15,0.68$ & 0.93 & $0.56,1.55$ \\
\hline Model 4 & 1.92 & $1.01,3.64$ & 0.32 & $0.15,0.68$ & 0.88 & $0.53,1.48$ \\
\hline Model 5 & 1.69 & $0.89,3.20$ & 0.34 & $0.16,0.72$ & 0.91 & $0.54,1.52$ \\
\hline Sensitivity $A^{b}$ & 2.20 & $0.87,5.61$ & 0.21 & $0.08,0.54$ & 1.62 & $0.86,3.04$ \\
\hline Sensitivity B & 1.00 & $0.33,3.02$ & 0.86 & $0.22,3.33$ & 0.96 & $0.34,2.67$ \\
\hline
\end{tabular}

${ }^{a}$ Model 1: Adjusted for maternal age, insurance status, gestational age at delivery and pregnancy history

Model 2: Adjusted for all variables in Model 1, preexisting diabetes, hypertension and pre-pregnancy obesity

Model 3: Adjusted for all variables in Model 2, trimester of entry into prenatal care and chart-documented hospitalizations during pregnancy

Model 4: Adjusted for all variables in Model 3, marital status, receipt of wages (by any household member) and education

Model 5: Adjusted for all variables in Model 4, smoking status during pregnancy and lifetime illicit drug use

${ }^{b}$ A: Model 5 restricted to women on Medicaid or without insurance

B: Model 5 restricted to nulliparous women who had experienced no previous pregnancy losses 
Table 4 Association between maternal race and stillbirth in models with interaction terms for WIC $x$ maternal race, stratified by WIC status

\begin{tabular}{|c|c|c|c|c|}
\hline \multirow[b]{2}{*}{ Model } & \multicolumn{2}{|c|}{$W I C=0$ (not enrolled $)$} & \multicolumn{2}{|c|}{ WIC = 1 (enrolled) } \\
\hline & Odds Ratio & $95 \% \mathrm{Cl}$ & Odds Ratio & $95 \% \mathrm{Cl}$ \\
\hline \multicolumn{5}{|l|}{ Model $1^{a}$} \\
\hline Black vs. White & 4.12 & $2.11,8.08$ & 0.59 & $0.27,1.29$ \\
\hline Hispanic vs. White & 1.67 & $1.02,2.73$ & 0.82 & $0.44,1.56$ \\
\hline \multicolumn{5}{|l|}{ Model 2} \\
\hline Black vs. White & 3.84 & $1.98,7.42$ & 0.58 & $0.26,1.27$ \\
\hline Hispanic vs. White & 1.85 & $1.14,3.02$ & 0.81 & $0.43,1.55$ \\
\hline \multicolumn{5}{|l|}{ Model 3} \\
\hline Black vs. White & 3.82 & $1.97,7.41$ & 0.57 & $0.26,1.28$ \\
\hline Hispanic vs. White & 1.80 & $1.10,2.95$ & 0.78 & $0.41,1.50$ \\
\hline \multicolumn{5}{|l|}{ Model 4} \\
\hline Black vs. White & 3.51 & $1.78,6.92$ & 0.59 & $0.27,1.31$ \\
\hline Hispanic vs. White & 1.59 & $0.94,2.66$ & 0.73 & $0.37,1.43$ \\
\hline \multicolumn{5}{|l|}{ Model 5} \\
\hline Black vs. White & 3.38 & $1.70,6.71$ & 0.67 & $0.30,1.51$ \\
\hline Hispanic vs. White & 1.68 & $0.99,2.85$ & 0.90 & $0.46,1.77$ \\
\hline \multicolumn{5}{|l|}{ Sensitivity $A^{b}$} \\
\hline Black vs. White & 4.78 & $1.67,13.69$ & 0.46 & $0.17,1.27$ \\
\hline Hispanic vs. White & 1.12 & $0.44,2.82$ & 0.82 & $0.38,1.80$ \\
\hline \multicolumn{5}{|l|}{ Sensitivity B } \\
\hline Black vs. White & 1.43 & $0.42,4.91$ & 1.23 & $0.34,4.50$ \\
\hline Hispanic vs. White & 2.20 & $0.90,5.39$ & 2.11 & $0.57,7.74$ \\
\hline
\end{tabular}

${ }^{a}$ Model 1: Adjusted for maternal age, insurance status, gestational age at delivery and pregnancy history

Model 2: Adjusted for all variables in Model 1, preexisting diabetes, hypertension and pre-pregnancy obesity

Model 3: Adjusted for all variables in Model 2, trimester of entry into prenatal care and chart-documented hospitalizations during pregnancy

Model 4: Adjusted for all variables in Model 3, marital status, receipt of wages (by any household member) and education

Model 5: Adjusted for all variables in Model 4, smoking status during pregnancy and lifetime illicit drug use

${ }^{b}$ A: Model 5 restricted to women on Medicaid or without insurance B: Model 5 restricted to nulliparous women who had experienced no previous pregnancy losses

compared to non-participants, there was very little association among White WIC participants [7]. The authors suggest that this may be due to the high rate of smoking among White WIC participants. WIC enrollment was also associated with a slightly higher smoking relapse rate among White Women compared to Black women [18]. While we controlled for both smoking during pregnancy and lifetime illicit drug use, which in our data were associated with WIC enrollment among White but not Black or Hispanic women, this did not change the observation that WIC appeared protective among Black women and was associated with stillbirth among White women. This positive association may be due to unmeasured confounding among the White women. A much lower proportion of White women compared to Black women participated in WIC ( $20 \%$ vs. $53 \%$ among live born controls), and it is likely that White women who participated in WIC were systematically different from White women who did not, with those who participated being at greater risk of stillbirth. Additionally, the positive association between WIC and stillbirth is substantially reduced when gestational age is not included in the models. In other words, the positive association between WIC and stillbirth among White women exists only within categories of gestational age. It has been suggested that racial/ethnic differences in induction of labor may be partly responsible for the racial disparity in stillbirth [19]. Within categories of gestational age, White women at risk of stillbirth and who are not on WIC may be more likely to be induced or have aggressively managed deliveries due to a combination of clinical indicators and demographic factors, compared to White women participating in WIC.

The protective association between WIC and stillbirth among Black women may also be due to residual confounding, where Black women who choose to participate in WIC are more health-conscious or proactive in addressing stillbirth risk factors than Black women who do not participate in WIC. Alternatively, WIC may be most beneficial to women with the specific risk factors for stillbirth, and Black women may represent this group due to a combination of preexisting conditions and access to medical care. Many factors have been shown to contribute, at least in small part, to the racial disparity in stillbirth [20]. Black and Hispanic women are more likely than White women to enter pregnancy with chronic conditions that increase the risk of stillbirth, including diabetes, hypertension, obesity and autoimmune disorders [21]. Overall, maternal health conditions contribute more to the hazard of stillbirth among Black women than among White and Hispanic women [22]. In our study, greater proportions of Black women who were obese, had diabetes or chronic hypertension enrolled in WIC compared to their White counterparts. For these Black women at a particularly high risk of stillbirth, the nutritional and social support that WIC provided may have contributed to averting a stillbirth, and one could speculate that we might have seen a similar association among White women had more high-risk White women participated in WIC prenatally. While the exact pathways through which WIC is associated with positive outcomes are unknown, evidence suggests it may be through favorable gestational weight gain patterns and better connections to medical services, both of which may serve to improve outcomes among women with chronic conditions [23].

The sensitivity analyses demonstrated that Medicaid status did not account for the supposed differential 
sorting of Black and White women into WIC, nor did restricting the study sample to women who had delivered at 37 weeks' gestation or later. However, racial/ ethnic differences in the association between WIC participation and stillbirth were eliminated when the analysis was restricted to nulliparous women with no previous losses, and all associations were pulled toward the null. Any nulliparity and multiparity with previous losses are associated with greater odds of stillbirth compared to women who have already experienced pregnancies without losses [13]. Further restricting to nulliparous women with no losses also excludes women who enrolled in WIC because they met the criteria for nutritional risk because they experienced previous losses [1]. Therefore, restricting to women who are all in their first pregnancy may represent a particularly homogenous group with fewer unmeasured confounders.

Our study had several important limitations. First, we did not have information on which participants in our study were actually eligible for WIC. However, we attempted to address this issue in our sensitivity analysis, which mirrored our original results. Several other studies have also addressed this issue by restricting their study sample to women receiving Medicaid [8, 9]. Second, we lacked information on timing of WIC enrollment and redemption of vouchers. Over half of pregnant women enrolling in WIC do so in the first trimester [1], and there was no evidence to suggest that timing of enrollment differs by race/ethnicity. We also attempted to reduce gestational age bias, where pregnancies with a longer gestation are less likely to result in stillbirth and have more time to enroll in WIC prenatally, by controlling for gestational age at delivery, though this can be problematic [24]. We were unable to use a true fetuses-at-risk approach as we did not have information on timing of WIC enrollment and could not construct exposed and unexposed cohorts, though we did attempt to approximate this analysis by not controlling for gestational age at delivery. A study that used a fetuses-at-risk approach showed that WIC participation was associated with favorable birth outcomes depending on the gestational week of enrollment [9]. We were also limited in our ability to precisely estimate some associations. In the sensitivity analyses which further restricted sample sizes, the confidence intervals were particularly wide. Finally, the question we used to determine WIC participation referred to the woman's sources of household income in the prior 12 months, so there was the possibility that women who responded affirmatively were not enrolled prenatally during the index pregnancy, but were rather enrolled because they were breastfeeding or in the postpartum period from a prior pregnancy. However, we did not note any significant differences in WIC participation by pregnancy history for any race/ethnicity.
Important strengths of this study included the use of a population-based dataset that was designed to examine stillbirth, a relatively rare birth outcome. As such, our study is one of only a handful of studies that specifically examine the association between WIC and stillbirth. We also were able to include selected clinical data in our analyses.

\section{Conclusions}

Our study suggests that WIC participation is associated with decreased risk of stillbirth among Black women. We believe WIC enrollment would be helpful for all WIC-eligible women, and that the null associations found for White and Hispanic women in this study are likely due to additional unmeasured factors associated with participation among these women.

\section{Additional file}

Additional file 1: "Weighted characteristics by race, and percent of each subgroup enrolled in WIC" provides the proportion of women with certain demographic characteristics participating in WIC. Proportions are presented separately by race/ethnicity. (DOCX $25 \mathrm{~kb}$ )

\section{Abbreviations}

BMI: Body mass index; OR: Odds ratio; WIC: Special Supplemental Nutrition Program for Women, Infants and Children

\section{Acknowledgements}

We would like to acknowledge all of the women who participated in this study.

\section{Funding}

The design of and data collection for the SCRN study was supported by grant funding from the Eunice Kennedy Shriver National Institute of Child Health and Human Development, National Institutes of Health, with supplemental funding from the Office of Research in Women's Health, National Institutes of Health (grants U10- HD045953 (Brown University), U10HD045925 (Emory University), U10-HD045952 (University of Texas Medical Branch at Galveston), U10-HD045955 (University of Texas Health Science Center at San Antonio), UK10-HD045944 (University of Utah Health Sciences Center), and U10-HD045954 (RTI International)). Funding did not support the analysis, interpretation or drafting of this manuscript.

\section{Availability of data and materials}

The datasets analyzed in this study are available from the Stillbirth Collaborative Research Network on reasonable request.

\section{Authors' contributions}

MA conceived and conducted the analyses and drafted the manuscript. $\mathrm{CH}$ assisted with the analysis concept and writing the manuscript. VT assisted with the analysis concept and construction of variables. $C H, D D, R G, R S, H P$, $\mathrm{VT}$ and BS all were part of the Stillbirth Collaborative Research Network (SCRN). They conceived and implemented the original case-control study from which the data used in this analysis were collected and all reviewed and provided feedback on the manuscript. CDB reviewed the original concept and manuscript and provided major feedback. All authors approved the final version of the manuscript.

\section{Ethics approval and consent to participate}

The study was approved by the institutional review boards of the academic centers and the participating hospitals: Brown University School of Medicine (Women \& Infants' Hospital of Rhode Island, Memorial Hospital of Rhode Island, Landmark Medical Center, Westerly Hospital, Caritas Good Samaritan 
Medical Center, Charlton Memorial Hospital, St. Luke's Hospital, Tobey Hospital, Kent Hospital, Newport Hospital, South County Hospital, Morton Hospital and Medical Center, Sturdy Memorial Hospital, Brockton Hospital), Emory University School of Medicine (Crawford Long Hospital, Grady Memorial Hospital, DeKalb Medical Center, DeKalb Medical Center at Hillandale, Piedmont Hospital, Atlanta Medical Center, Northside Hospital), University of Texas Medical Branch at Galveston (University of Texas Medical Branch at Galveston, Clear Lake Regional Medical Center, Mainland Medical Center, Woman's Hospital of Texas, Brazosport Memorial Hospital, Christus St. Joseph Hospital, Christus St. John Hospital, Hermann Hospital, Memorial Hermann Southeast Hospital, Methodist Hospital, Angleton Danbury Medical Center, St. Luke's Episcopal Hospital), University of Texas Health Science Center at San Antonio (Methodist Hospital, Methodist Metropolitan Hospital, University Hospital, Baptist Medical Center, North Central Baptist Hospital, North East Baptist Hospital, Southeast Baptist Hospital, St. Luke's Baptist Hospital, Southwest General Hospital, Christus Santa Rosa Hospital, Wilford Hall Medical Center (59th Medical Wing)), University of Utah School of Medicine (Alta View Hospital, Cottonwood Hospital, Jordan Valley Hospital, LDS Hospital, Pioneer Valley Hospital, St. Mark's Hospital, Salt Lake Regional Medical Center, University Hospital, North Davis Hospital, American Fork Hospital, Orem Community Hospital, Utah Valley Regional Medical Center, McKay-Dee Hospital, Ogden Regional Hospital, Intermountain Medical Center) and the data coordinating center, RTI International. All participants in the case-control study gave written informed consent.

\section{Consent for publication}

Not applicable.

\section{Competing interests}

The authors declare that they have no competing interests.

\section{Publisher's Note}

Springer Nature remains neutral with regard to jurisdictional claims in published maps and institutional affiliations.

\section{Author details}

'Department of Epidemiology, Rollins School of Public Health, Emory University, 1518 Clifton Road, Atlanta, GA 30322, USA. ${ }^{2}$ Biostatistics and Epidemiology Division, RTI International, Research Triangle Park, North, Carolina, USA. ${ }^{3}$ Department of Obstetrics and Gynecology, University of Virginia School of Medicine, Charlottesville, Virginia, USA. ${ }^{4}$ Department of Obstetrics and Gynecology, Columbia University Medical Center, New York, NY, USA. ${ }^{5}$ Division of Maternal-Fetal Medicine, Department of Obstetrics and Gynecology, School of Medicine, University of Utah, Salt Lake City, UT, USA. ${ }^{6}$ McGovern Medical School, University of Texas Health Science Center, Houston, TX, USA. ${ }^{7}$ The Warren Alpert School of Medicine, Brown University, Providence, RI, USA.

Received: 13 November 2017 Accepted: 26 June 2018

Published online: 24 July 2018

\section{References}

1. Thorn B, Tadler C, Huret N, Trippe C, Ayo E, Mendelson M, Patlan KL, Schwartz G, Tran V: WIC Participant and Program Characteristics 2014. Alexandria, VA: U.S. Department of Agriculture, Food and Nutrition Service.; 2015

2. Besharov DJ, Germanis P. Evaluating WIC. Eval Rev. 2000;24(2):123-90.

3. Joyce $T$, Gibson $D$, Colman $S$. The changing association between prenatal participation in WIC and birth outcomes in new York City. J Policy Anal Manage. 2005;24(4):661-85.

4. Joyce T, Racine A, Yunzal-Butler C. Reassessing the WIC effect: evidence from the pregnancy nutrition surveillance system. J Policy Anal Manage. 2008;27(2):277-303.

5. Panel to Evaluate the USDA's Methodology for Estimating Eligibility and Participation for the WIC Program. Estimating eligibility and participation for the WIC program: final report. In: Ver Ploeg M, Betson DM, editors. In. Washington: D.C.; 2003.

6. Committee on Scientific Evaluation of WIC Nutrition Risk Criteria Food and Nutrition Board. WIC nutrition risk criteria: a scientific assessment in. Washington, D.C.: institute of. Medicine. 1996;
7. Khanani I, Elam J, Hearn R, Jones C, Maseru N. The impact of prenatal WIC participation on infant mortality and racial disparities. Am J Public Health. 2010;100(Suppl 1):S204-9.

8. Sonchak L. The impact of WIC on birth outcomes: new evidence from South Carolina. Matern Child Health J. 2016;20(7):1518-25.

9. Fingar KR, Lob SH, Dove MS, Gradziel P, Curtis MP. Reassessing the association between WIC and birth outcomes using a fetuses-at-risk approach. Matern Child Health J. 2017;21(4):825-35.

10. El-Bastawissi AY, Peters R, Sasseen K, Bell T, Manolopoulos R. Effect of the Washington special supplemental nutrition program for women, infants and children (WIC) on pregnancy outcomes. Matern Child Health J. 2007;11 (6):611-21.

11. Keene Woods N, Reyes J, Chesser A. Infant mortality and race in Kansas: associations with women, infants, and children services. J Prim Care Community Health. 2016;7(3):194-8.

12. MacDorman MF, Gregory EC. Fetal and perinatal mortality: United States, 2013. Natl Vital Stat Rep. 2015;64(8):1-24.

13. Stillbirth Collaborative Research Network Writing G. Association between stillbirth and risk factors known at pregnancy confirmation. JAMA. 2011; 306(22):2469-79.

14. Parker CB, Hogue CJ, Koch MA, Willinger M, Reddy UM, Thorsten VR, Dudley DJ, Silver RM, Coustan D, Saade GR, et al. Stillbirth collaborative research network: design, methods and recruitment experience. Paediatr Perinat Epidemiol. 2011;25(5):425-35

15. Joseph KS, Kramer MS. The fetuses-at-risk approach: survival analysis from a fetal perspective. Acta Obstet Gynecol Scand. 2018;97(4):454-65.

16. Liu CH, Liu H. Concerns and structural barriers associated with WIC participation among WIC-eligible women. Public Health Nurs. 2016;33(5):395-402.

17. Black MM, Cutts DB, Frank DA, Geppert J, Skalicky A, Levenson S, Casey PH, Berkowitz C, Zaldivar N, Cook JT, et al. Special supplemental nutrition program for women, infants, and children participation and infants' growth and health: a multisite surveillance study. Pediatrics. 2004;114(1):169-76.

18. Yunzal-Butler $C$, Joyce $T$, Racine AD. Maternal smoking and the timing of WIC enrollment. Matern Child Health J. 2010;14(3):318-31.

19. Singh J, Reddy UM, Huang CC, Driggers RW, Landy HJ, Grantz KL. Racial/ ethnic differences in labor induction in a contemporary US cohort: a retrospective cohort study. Am J Perinatol. 2018;35(4):361-8.

20. Hogue CJ, Silver RM. Racial and ethnic disparities in United States: stillbirth rates: trends, risk factors, and research needs. Semin Perinatol. 2011:221-33.

21. Fridman M, Korst LM, Chow J, Lawton E, Mitchell C, Gregory KD. Trends in maternal morbidity before and during pregnancy in California. Am J Public Health. 2014;104(Suppl 1):S49-57.

22. Willinger M, Ko CW, Reddy UM. Racial disparities in stillbirth risk across gestation in the United States. Am J Obstet Gynecol. 2009;201(5):469 e461-8.

23. Currie J, Rajani I. Within-mother estimates of the effects of WIC on birth outcomes in new York City. Econ Inq. 2015;53(4):1691-701.

24. Wilcox AJ, Weinberg CR, Basso O. On the pitfalls of adjusting for gestational age at birth. Am J Epidemiol. 2011;174(9):1062-8.

\section{Ready to submit your research? Choose BMC and benefit from:}

- fast, convenient online submission

- thorough peer review by experienced researchers in your field

- rapid publication on acceptance

- support for research data, including large and complex data types

- gold Open Access which fosters wider collaboration and increased citations

- maximum visibility for your research: over $100 \mathrm{M}$ website views per year

At BMC, research is always in progress.

Learn more biomedcentral.com/submissions 